\title{
Seamless Handover in Buildings Using HVAC Ducts: A New System Architecture
}

\author{
Ariton E. Xhafa, Paisarn Sonthikorn, Ozan K. Tonguz, Pavel V. Nikitin ${ }^{\dagger}$, Ahmet G. Cepni, Daniel D.Stancil, \\ Benjamin Henty,and Dagfin Brodtkorb ${ }^{\ddagger}$ \\ Carnegie Mellon University, Dept. of Electrical and Computer Engineering, \\ Pittsburgh, PA 15213-3890, USA \\ †University of Washington, Department of Electrical Engineering \\ Seattle, WA 98195-2500, USA \\ ${ }^{\ddagger}$ Asea Brown Boveri Corporate Research, Bergerveien 12, P.O.Box 90 \\ N-1735 Billingstad, NORWAY
}

\begin{abstract}
In this paper, we present an innovative solution to the handover problem in multi-story buildings using HVAC ducts for wireless communications. The proposed solution is based on a new system architecture design of the indoor wireless networks that use the heating, ventilation, and air conditioning (HVAC) ducts as a communication channel. Exploiting the system architecture design and the time diversity at the mobile terminal, we show that intra-floor handover can be eliminated, while interfloor handover can be substantially reduced due to the fact that multiple floors can be served via the same access point. Our results indicate that the mean number of handovers per call achieved in the indoor wireless networks that use HVAC ducts reduces; e.g., by up to a factor of nine for the illustrative example considered in this paper compared to that of indoor wireless networks that do not use HVAC ducts. The improvement; i.e., reduction in the mean number of handovers in multi-story building, depends on the number of access points used.
\end{abstract}

\section{INTRODUCTION}

One of the goals of the next generation wireless networks is to provide high-speed internet access in buildings. While traditional indoor wireless communication systems transmit and receive signals via a network of transmitters, receivers, and antennas placed throughout the interior of a building [1], an alternative approach is to use heating, ventilation, and air conditioning ducts as a communication channel [2]. Regardless of the approach used to transmit and receive signals in indoor wireless networks (i.e., traditional or HVAC systems), the small cell size of the indoor wireless networks results in a high number of handovers. To ensure service continuity, handover management remains one of the most important system issues that needs to be addressed.

Handover in indoor wireless networks was first reported in [3], where the author concluded that handover is a necessary feature in wireless office systems. This was based on 1991 field trials that were performed in an indoor wireless system that was based on CT3 technology that used Multi Carrier/ Time Division Multiple Access/ Time Division Duplex (MC/TDMA/TD) and operated at $900 \mathrm{MHz}$ band [3]. Since then, numerous research papers and studies have investigated the issue of handover in indoor wireless networks [4]-[11]. The studies reported in [4]-[7] apply well-known techniques and ideas from the outdoor wireless networks to investigate and improve the handover performance of the indoor wireless networks. The drawback of these studies was that user movements were confined to two dimensions, while it is a wellknown fact that user movements in a multi-story building can occur in three dimensions. For the first time, the impact of 3 dimensional (3-D) user movements in handover performance of indoor wireless networks was investigated in [8]-[10], where the authors modeled 3-D user movement including vertical motions through staircases and via elevators in highrise buildings. Even though the aforementioned work deals with handover issues in indoor wireless networks, to date, handover issue for an indoor wireless network that utilizes the new HVAC duct technology has not been studied.

In this paper, a new system architecture is proposed for indoor wireless networks that use HVAC ducts and the handover issue in such networks is investigated. Exploiting the proposed system architecture and the time diversity at the mobile terminal, we show that intra-floor handovers can be eliminated, while inter-floor handovers can be substantially reduced due to the fact that multiple floors can be served via the same access points.

Our results show that compared to indoor wireless networks that do not use HVAC ducts, the mean number of handovers per call can be reduced by up to a factor of nine in the HVAC indoor wireless networks (HVAC-IWN) for the illustrative scenario investigated in this paper.

The remainder of the paper is organized as follows. The background and motivation are described in Section II. An HVAC-IWN system description is given in Section III. In Section IV we investigate the handover issue in HVAC-IWN and illustrate our approach via an example in Section V. In Section VI we discuss the implications of our findings. Finally, conclusions are drawn in Section VII.

\section{BACKGROUND AND MOTIVATION}

Previous theoretical work showed that indoor wireless networks using HVAC ducts can support data rates in excess of $1 \mathrm{Gbps}$ for distances up to $500 \mathrm{~m}$ in $2.4 \mathrm{GHz}$ ISM band [12]. Experiments using actual synthesized signals through a building HVAC duct system demonstrate the ability to transmit with a spectral efficiency of $3.74 \mathrm{bps} / \mathrm{Hz}$, corresponding to 
over $300 \mathrm{Mbps}$ transmission rate within $2.4 \mathrm{GHz}$ ISM band [13]. The process of designing an indoor wireless network that uses HVAC ducts is an ongoing project at Carnegie Mellon University. While physical layer issues, such as propagation, channel characteristics, capacity, link budget calculations [14], etc., have been studied extensively, to date; there is no published work that treats the handover issue in HVAC-IWN.

The main objective of this paper is to propose a system design architecture that yields seamless communications in HVAC-IWN.

Next, we describe the proposed system architecture for HVAC-IWN.

\section{Proposed System ARCHItecture OF HVAC-IWN}

An alternative approach to providing indoor wireless communications in buildings is to propagate the RF signals through the heating, ventilation and air conditioning ducts. Concepts and preliminary work on this subject have been reported in [2].

Figure 1 illustrates a typical HVAC system. The indoor wireless network that uses the HVAC ducts are connected to the outside IP-network via the access point controller (APC). The APC serves the whole building and is connected to one or more antennas in the duct. Each antenna acts as a remote antenna (RA) for a particular floor, or for a group of floors. Figure 1 shows an APC connected to an RA that serves three floors in a building. In this model, each receiving antenna in a room/office is placed in the HVAC duct that is located in its corresponding room/office. The RF signal sent from the RA propagates through the ducts and is received by the receiving antennas placed at each room/office.

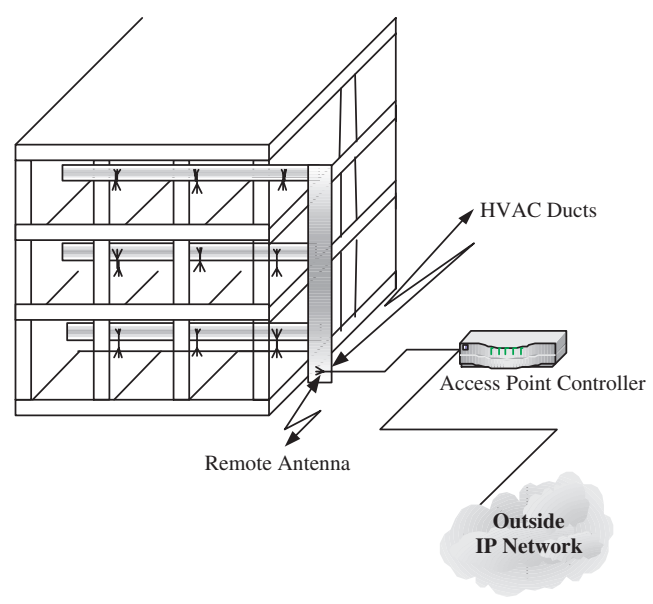

Fig. 1. An HVAC system example.

Figure 2 illustrates the building blocks for the APC. The APC consists of the End User Mobile Switch (EUMS) and access points (APs). The EUMS interfaces with the outside network, and controls and manages the APs. An AP transmits and receives signals through the corresponding RA via a coaxial cable. An AP communicates to another AP via the EUMS module. An RA is responsible for one or more floors. All the intelligence and control is in the APC; hence, the RAs simply act as repeaters. The APC can connect to more than one RA. For example, the third floor might require a separate
RA, while the first and the second floor in the given scenario (see Figure 1) are still served by the same RA. In this case, an RA can be placed at the beginning of the duct in the third floor.

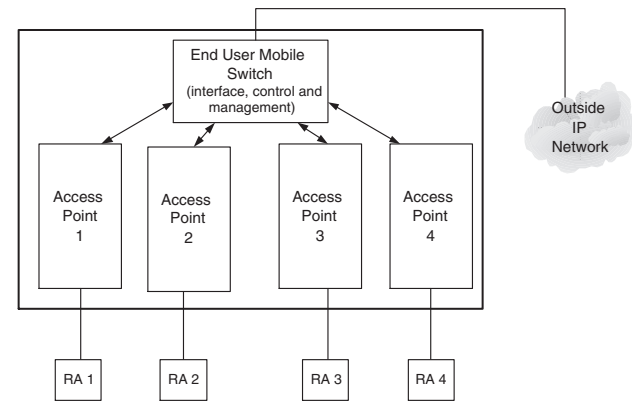

Fig. 2. A reference model for the APC.

Figure 3 shows how indoor wireless networks will operate on a given floor. The connection to the terminals can be done i) via a wireless link, i.e., a receiving antenna can be mounted in the duct and another one can be used to couple the signal and transmit it in the office; or ii) a louver might be present at the office, in which case an antenna mounted in the duct will not be necessary; or iii) via a cable, i.e., again a receiving antenna can be mounted in the duct and the terminal can be connected to this antenna via a cable.

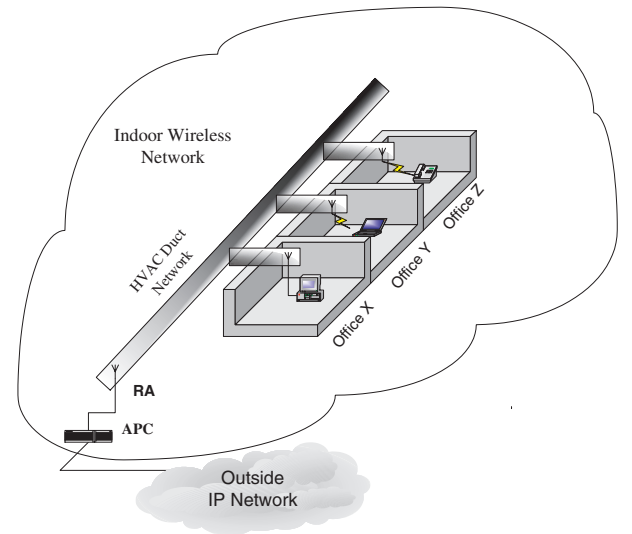

Fig. 3. Indoor wireless network using HVAC ducts in a floor.

In the next section, we utilize the proposed system architecture design to deal with the handover issue in HVAC-IWN.

\section{HANDOVER IN HVAC-IWN}

In an indoor wireless network environment, limited wireless resources and user mobility require effective frequency planning in order to minimize the handover call dropping without compromising network utilization. In a HVAC-IWN intra-floor and inter-floor handover can be reduced drastically; hence, handover call dropping probability reduces drastically. To show this, next we consider intra- and inter-floor handovers in HVAC-IWN.

\section{A. Intra-floor Handover}

Consider the user movements on the floor given in Figure 3. Assume that Offices $\mathrm{X}, \mathrm{Y}$, and $\mathrm{Z}$ are served by different access 
points, which would be the case in traditional indoor wireless networks. Furthermore, assume that the user starts the call at Office $\mathrm{X}$ and while the call is in progress, the user moves toward Office Z. Since each office is served by a different AP, a handover is needed when the user moves from the coverage area of one AP to the other. In our scenario, a handover is needed when the user moves from Office $\mathrm{X}$ to Office $\mathrm{Y}$ and another handover is needed when the user moves from Office Y to Office Z. In the case of HVAC-IWN, all three offices are served by the same RA; hence, the same AP. Therefore, handovers do not occur as the user moves from Office $\mathrm{X}$ to Office Z. The same argument can be made for the rest of the floor, not just for the three offices shown in Figure 3. Thus, the whole floor can be covered by a single RA; hence, a single AP. The reason for this is the low attenuation that the RF signal experiences in HVAC ducts. Thus, the RF signal transmitted from the RA can "reach" any office/room that are in the same floor with the RA. As the user moves from Office $X$ to Office $Z$, a frequency change might be necessary, because the received RF signal suffers from multipath and reflections that occurs in the ducts, which can create deep nulls and oscillating behavior in the channel frequency response. However, the connection that the user has with AP is moved from one channel to another within the same access point, AP; hence, there is no need for renegotiating the existing quality of service (QoS) for the user since handover occurs within the same access point AP.

Due to low attenuation of RF signals in HVAC ducts, we anticipate that one or more floors are served by at most one RA; hence, the same AP. Thus, the aforementioned handover procedure is within the AP module serving this particular floor and is done without the need for renegotiating QoS for the mobile users moving on the floor. As a result, each floor can be considered as one big cell served by the same AP; hence, no handover is needed between two APs when the mobile user moves on the same floor.

The intra-floor handovers within the same AP can be eliminated via the use of diversity at the mobile terminal or RA. For example, link improvement can be achieved via time diversity. Such improvement implies that the frequency channel that the user was using at Office $\mathrm{X}$ is available throughout the floor. Hence, the mobile user can use the same frequency channel (range) even when the mobile user moves to anywhere on the same floor. As a result, even the switching load (i.e., handover from one channel or frequency range to another) within the same AP is eliminated.

The approach described above deals with two dimensional (2-D) mobile user movement. However, in indoor wireless networks, one needs to consider 3-D user movements, staircase and elevator movements and, hence, inter-floor handover. Next, we investigate the inter-floor handover in HVAC-IWN.

\section{B. Inter-floor Handover}

Inter-floor handover can occur because of: i) movement of mobile users between the floors through staircases; and ii) movement of mobile users between the floors via the elevator. To deal with inter-floor handover in HVAC-IWN networks we propose the following:

Staircases between the floors are covered by receiving antennas that use the same frequency range as the one used in the respective floors. Assume that a mobile user moves through the staircases from the first floor to the second floor. The following scenarios could occur:

- Both floors are served by the same RA/AP; hence, a handover is not needed, since the mobile user can use the same frequency range in the visiting floor. Note that an AP and its RA(s) can serve at least one floor.

- Both floors are served by different APs; hence, a soft handover takes place between the APs.

Since the EUMS at the APC takes care of the interfloor handover process in HVAC-IWN, the handover is fast. The handover failure is also low since APC controls all the resources in the HVAC-IWN.

In case of elevator movement, what we propose is similar to the idea proposed in [10]. However, instead of an APC at the elevator ceiling, we propose to use an RA at the elevator ceiling. The RA is connected to the AP that is designed to serve the elevator cells only; however, another option would be that the RA is connected to an AP that already serves a floor or multiple floors. In the latter case, inter-floor handovers can be reduced drastically, since no handover occurs when a mobile user moves through the elevator cells that are served by the same AP that was already serving him/her. In the earlier case; i.e., one AP for elevator cells only, the whole frequency range is available in this elevator cell. Assume that a mobile user moves from a particular floor, say the third floor (which is assumed to be served by AP3) and goes down to the second floor (which is assumed to be served by AP2) using the elevator. At the time that the user enters the elevator, a handover takes place and the user is transferred from the RA serving the third floor to the RA serving the elevator. At the APC this means that a handover takes place between AP3 and AP4 (we assume that AP4 is used to connect to the RA at the elevator ceiling). As the elevator moves to the second floor, after the door is open, the user in the elevator experiences another handover. At the APC, this translates to a handover between AP4 and AP2. As one can see, since the whole frequency band is available in the elevator, a soft handover could take place all the time.

To illustrate the impact of HVAC-IWN design on the handover rate, in the next section, an illustrative example is provided.

\section{AN ILlustrative EXAMPLE}

In order to illustrate the impact of HVAC system design on the handover issue, we consider a similar scenario like the one described in Section II.B in [9]. Figure 4 illustrates this scenario. The floor consists of 9 cells, where the grey region is the staircase region, thus the staircase region (cell), and the rest of the cells ( 8 of them) make up the floor region. Movements of mobile users on the floor are confined to the planar movement, i.e., 2-D movement, if the mobile users are not in the staircase region. If the mobile users are in the staircase region, their movement could be on the same floor, or they could move in vertical directions, either to the floor above or below. Therefore, a handover will be required whenever a user moves from one cell to another cell on the floor.

Figure 5 illustrates the concept of the HVAC-IWN for the same scenario as the aforementioned one. Observe that the 


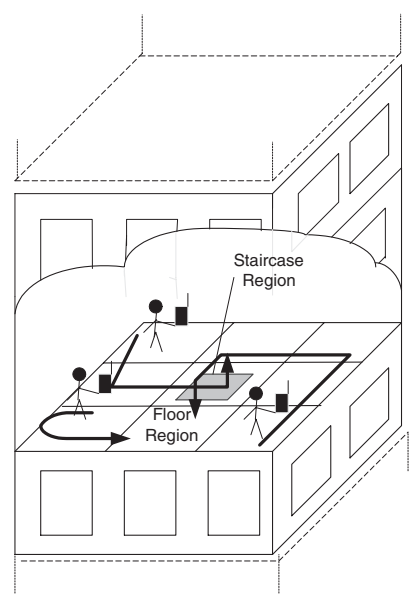

Fig. 4. The scenario described in [9].

number of cells that make up the floor region is one; hence, eliminating handovers between different cells that exist in the floor region. Therefore, the intra-floor handovers are reduced drastically. However, the inter-floor handovers still occur in HVAC-IWN, since the staircase region is served by a different AP than the rest of the floor.

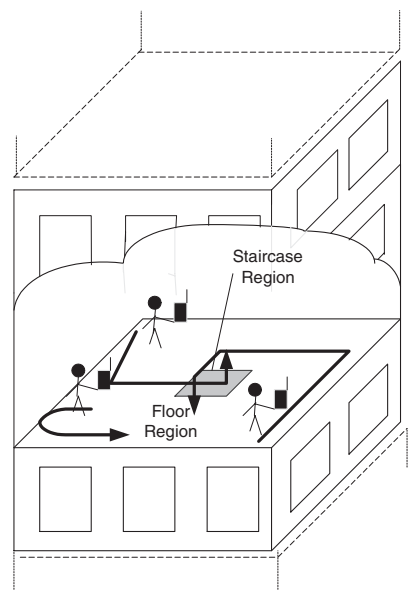

Fig. 5. The concept of HVAC-IWN network.

To quantify the difference in the performance between the two approaches described in Figures 4 and 5, we calculate the mean number of handovers per call under the following assumptions: each floor in the building is $60 \mathrm{~m} \times 60 \mathrm{~m} \times 9$ $\mathrm{m}$; the call duration is assumed to be exponentially distributed with mean 500 seconds; next direction is determined randomly with probability $\alpha=0.7$ in horizontal direction, thus on the same floor, and $\beta=0.3$ in vertical direction, thus to another floor provided that the mobile user is in the staircase region (see Figure 4); horizontal and vertical speeds which are assumed to be constant in this paper, as opposed to uniformly distributed between [0,4] and [0,4/3] km/h as claimed in [9]. The mean number of handovers per call, $E[H]$, can be found from a modified version of Eqn.(6) in [9].

In Figure 6 we investigate the impact of $\beta$ on the mean number of handovers per call in HVAC-IWN and in the indoor wireless network that is not using HVAC ducts to transmit the RF signals. We assume that in the latter case, the floor is partitioned into nine cells, while in the case of indoor wireless networks using HVAC ducts, the floor is partitioned into two cells, the staircase region and the rest of the floor cells. It is clear from Figure 6 that as $\beta$ increases, the mean number of vertical motion handovers increases; as a result, the mean number of handovers per call increases. Another observation is that the mean number of handovers per call in the case of HVAC-IWN is much less than that of traditional indoor wireless networks. Even for high values of $\beta$, the mean number of handovers experienced by a call in progress is almost seven, which is approximately three less than the mean number of handovers that the call would experience in a traditional indoor wireless network. The reason why there are still handovers at $\beta=0$ is due to the fact that the call could start from a staircase region (the area of the staircase), and the user might move to one of the floor cells which are served by different APs than the staircase cell.

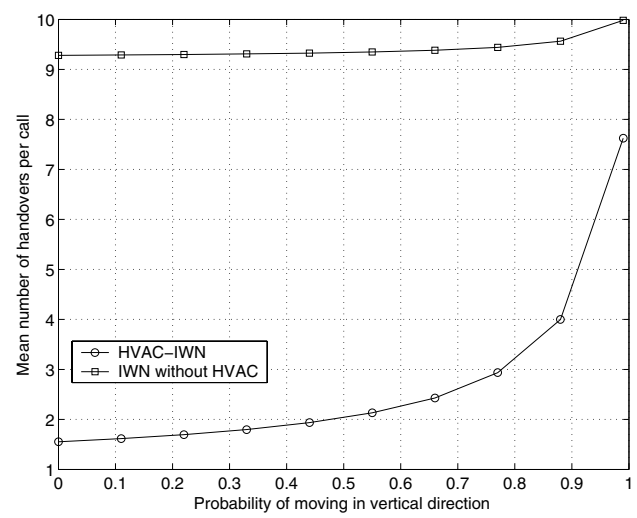

Fig. 6. The impact of vertical direction selection in the staircase region on the mean number of handovers.

From Figure 7, one can see that as the mean of the horizontal speed user movement increases, the mean number of handovers that a call in progress experiences increases. In the case of HVAC-IWN, there is the staircase region/ elevator region, and the rest of the floor is the gigantic cell. As the horizontal speed increases, the mobile users are more likely to be in the staircase/elevator region, and hence, there are more handovers. However, for HVAC-IWN this number is up to nine times less than that of traditional indoor wireless networks, which is a substantial improvement.

It is important to mention here that although a reduction by a factor of nine in the mean number of handovers was found for the aforementioned scenario, the benefits of using HVACIWN and the proposed system architecture varies. This will depend on the number of cells in the multi-story building. For example, if the number of cells in the multi-story building is greater than 9, then one would expect a higher reduction factor than the one reported in this paper. Further research is needed to quantify and investigate the dependency of the performance on the number of cells and other system parameters.

\section{Discussions}

The solution to the handover problem in HVAC-IWN is based on the proposed system architecture for these networks and time diversity at the mobile terminal. It was shown 


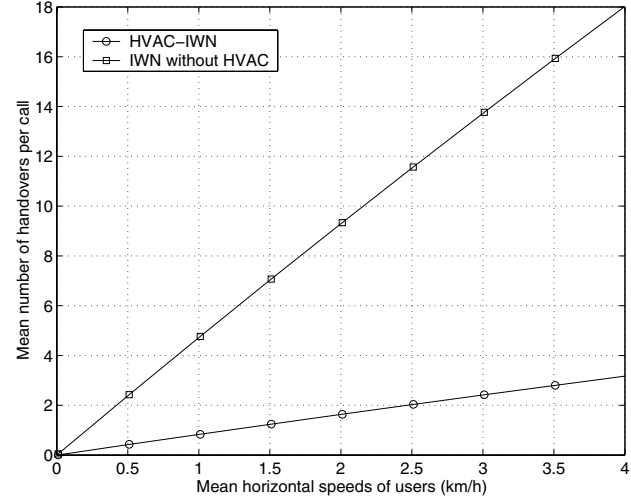

Fig. 7. The impact of horizontal speed user movement on the mean number of handovers.

that this system design and architecture eliminates intra-floor handover and improves significantly inter-floor handover rate.

However, there is a tradeoff between the improvement that one can achieve in the handover performance by using time diversity and the cost and complexity of using simple receivers that do not use time diversity. If time diversity is not used, then intra-floor handovers will occur within the same AP; hence, there is no need for renegotiating QoS for the mobile users. In case of traditional indoor wireless networks that do not use HVAC ducts, an intra-floor handover means renegotiating QoS, since the mobile users experiencing handover are served by different base stations (BSs). Hence, still the extra traffic introduced in the network due to handover is higher in the latter scenario.

Our results clearly show that the use of HVAC ducts in indoor wireless networks decreases the mean number of handovers that a call in progress experiences by up to a factor of nine, which is quite substantial. While the system design of HVAC-IWN is still an ongoing process at Carnegie Mellon University, it is clear that HVAC-IWN have a great potential not only in terms of the high capacity and coverage that they provide [2], [12], but also in providing a natural solution to the handover issue.

\section{CONCLUSIONS}

In this paper, we proposed a solution to the handover problem in indoor wireless networks. Our solution exploits the proposed system architecture design of the indoor wireless networks using heating, ventilation, and air conditioning ducts as a communication channel. Due to the low attenuation that $\mathrm{RF}$ signals experience in HVAC ducts, it is possible to use a single AP to cover at least one floor in multi-story buildings.

We show that using time diversity and single AP coverage, intra-floor handovers can be eliminated, while inter-floor handover can be treated as soft handovers in most scenarios.
Our results show that, for the illustrative scenario studied in this paper, the mean number of handovers that a call in progress experiences is reduced by up to a factor of nine compared to that of traditional indoor wireless networks that do not use HVAC ducts as a communication channel. This, once again shows the potential of indoor wireless networks that use HVAC duct instead of traditional wireless communication technology.

\section{REFERENCES}

[1] D. Molkdar, "Review on radio propagation into and within buildings", IEE Proc., vol. 138, no. 1, pp. 61-73, Feb. 1991.

[2] D. D. Stancil, O. K. Tonguz, A. Xhafa, A. Cepni, P. Nikitin, and D. Brodtkorb, "High-speed Internet access via HVAC ducts: A new approach", in Proc. of IEEE Global Telecomm. Conf. (GLOBECOM'01), vol. 6, pp. 3604-3607, San Antonio, Texas, Nov. 2001.

[3] G. K. Wolterin, "Handoff, a necessary feature in wireless office systems", 1st International Conference on Universal Personal Communications, 1992. ICUPC '92 Proceedings., pp. 13.06/1 -13.06/6, 29 Sept.Oct. $1,1992$.

[4] C. G. Zhang, H. M. Hafez, and D. D. Falconer, "Traffic handling capability of a broadband indoor wireless network using CDMA multiple access", IEEE Journal on Selected Areas in Communications, Vol. 12, no. 4, pp. 645-653, May 1994.

[5] P. Theodorou, J. Elmirghani, and R. Cryan, "Performance of ATM optical wireless LANs with fixed channel assignment", IEEE Global Telecommunications Conference (GLOBECOM '99), Vol. 1B, pp. 598602, 1999

[6] S. G. Choi, J. K. Choi, and H. W. Jung, "Design of Wireless Access Points with Multiple Radio Transceivers for In-Door ATM LAN Application", —em ICACT'99, 10-12 February 1999.

[7] S. Lu, R. Srikant, and V. Bharghavan, "Adaptive resource reservation for indoor wireless LANs", IEEE Global Telecommunications Conference (GLOBECOM '96) "Communications: The Key to Global Prosperity", Vol. 1, pp. 11-15, 18-22 Nov 1996.

[8] T. S. Kim, M. Y. Chung, and D. K. Sung, "Mobility and traffic analyses in three-dimensional PCS environments" IEEE Transactions on Vehicular Technology, Vol. 47, no. 2, pp. 537-545, May 1998.

[9] T. S. Kim, M. Y. Chung, and D. K. Sung, "Mobility modeling and traffic analysis in three-dimensional indoor environments", IEEE Transactions on Vehicular Technology, Vol. 47, no. 2, pp. 546-557, May 1998.

[10] T. S. Kim, M. Y. Chung, and D. K. Sung, "Moving elevator-cell system in indoor buildings" IEEE Transactions on Vehicular Technology, Vol. 49 , no. 5, pp. 1743-1751, Sep 2000.

[11] V. Di Claudio, E. Graziosi, E. Maurizio, F. Mecella, and F. Santucci, "A proposal for seamless handovers in GSM indoor wireless networks" IEEE Proceedings of Vehicular Technology Conference(VTC 2001 Spring), Vol. 4, pp. 2544-2548, 2001.

[12] A. Xhafa, O. K. Tonguz, A. Cepni, D. D. Stancil, P. Nikitin, and D. Brodtkorb, "Theoretical Estimates of HVAC Duct Channel Capacity for High-speed Internet Access", Proceedings of IEEE International Conference on Communications (ICC 2002), Vol. 2, pp. 936-939, New York, April 28-May 2, 2002.

[13] A. G. Cepni, A. E. Xhafa, P. V. Nikitin, D. D. Stancil, O. K. Tonguz, and D. Brodtkorb, "Multicarrier Signal Transmission through HVAC Ducts: Experimental Results for Channel Capacity", IEEE Proceedings of the Fall 2002 IEEE Vehicular Technology Conference (VTC Fall 2002), vol. 1, pp. 331-335, 2002.

[14] O. K. Tonguz, D. D. Stancil, A. E. Xhafa, A. G. Cepni, P. V. Nikitin, and D. Brodtkorb, "An empirical path loss model for HVAC duct systems", in Proc. IEEE Global Telecommunications Conference (GLOBECOM'02), vol. 2, pp. 1850-1854, Taipei, Taiwan, 17-21 Nov. 2002. 